\title{
Three-dimensional modelling of scattering loss in InGaAsP/InP and silica-on-silicon bent waveguides
}

Caterina Ciminelli
c.ciminelli@poliba.it

Vittorio M. N. Passaro

Francesco Dell'Olio

Mario N. Armenise
Dipartimento di Elettrotecnica ed Elettronica, Politecnico di Bari, Via Re David 200 - 70125 Bari, Italy

Dipartimento di Elettrotecnica ed Elettronica, Politecnico di Bari, Via Re David 200 - 70125 Bari, Italy

Dipartimento di Elettrotecnica ed Elettronica, Politecnico di Bari, Via Re David 200 - 70125 Bari, Italy

Dipartimento di Elettrotecnica ed Elettronica, Politecnico di Bari, Via Re David 200 - 70125 Bari, Italy

A three-dimensional (3D) method for the estimation of scattering loss due to sidewalls roughness in bent optical waveguides is proposed and validated. The approach, based on Volume Current Method (VCM), has been pointed out to accurately calculate the scattering loss as dependent on curvature radius and wavelength. An exponential model has been employed to analytically describe the sidewalls roughness and a 3D mode solver based on mode-matching method has been used to calculate optical field distribution in the bent waveguide cross-section.

Scattering loss suffered by two low index contrast waveguides has been investigated by the developed algorithm. For a buried InCaAsP/InP waveguide and a $6 \mu \mathrm{m} \times 6 \mu \mathrm{m}$ silica-on-silicon guiding structure scattering loss dependence on bending radius, wavelength, roughness, correlation length and standard deviation has been investigated and discussed. Because of the different index contrast values, InGaAsP/InP waveguide exhibits a scattering loss which is quite six times larger than in silica-on-silicon. For both guiding structures, quasi-TM mode shows a larger scattering loss than quasi-TE one. [DOI: 10.2971/jeos.2009.09015]

Keywords: scattering loss, sidewall roughness, bent waveguides, silica-on-silicon, indium phosphide

\section{INTRODUCTION}

Propagation loss estimation has a key role in optical waveguides characterization. Guiding structure loss often limits photonic devices performance and, then, analytical and numerical algorithms can be very useful to predict propagation loss exhibited by a specific waveguide.

Loss sources in the most common waveguides are material absorption and geometrical or physical discontinuities (as those induced by sidewalls roughness), which produce some radiated power. In bent waveguides, bending loss has to be taken into account too. Among different contributions to propagation loss, scattering loss due to sidewall roughness is the most difficult to estimate. Moreover, this contribution can be only minimized by appropriate design and technological strategies but it cannot be eliminated.

Scattering loss suffered by bent waveguides employed in integrated ring resonators imposes the ultimate limit for quality factor and finesse in these devices [1]. Consequently, it is crucial to be able to accurately estimate this loss contribution depending on waveguide fabrication process and geometry, bending radius and operating wavelength.

The calculation problem of scattering loss due to sidewall roughness has been firstly widely investigated in [2] by a two-dimensional (2D) analytical approach based on Coupled
Mode Theory (CMT). VCM application to this problem has been proposed in [3] and an analytic formula for scattering loss estimation in planar waveguides has been derived in [4]. In $[5,6]$, the application of $2 \mathrm{D}$ analysis results to rectangular waveguides has been proposed through the use of the wellknown effective-index method. Si-wire waveguides scattering loss has been approximately computed by this approach. Recently, two different 3D approaches have been developed for sidewall roughness induced loss estimation [7, 8]. In [7], CMT has been employed to calculate sidewall roughness induced coupling between guided and radiation modes (derived by an approximated semi-analytical method). In [8], VCM has been newly used to estimate scattering loss in silicon-on-insulator, silicon oxynitride $/ \mathrm{SiO}_{2}$ and $\mathrm{Si}_{3} \mathrm{~N}_{4} / \mathrm{SiO}_{2}$ rectangular waveguides. In all these papers only straight waveguides have been considered. A 2D algorithm developed for scattering loss estimation in micro-disk resonators has been reported in [9]. Another 2D approach based on the series expansion of roughness function has been adopted in [10] to estimate sidewalls roughness induced loss in a semiconductor ring laser designed to be included in a fully integrated active optical gyroscope.

In this paper, a 3D approach for scattering loss computation in bent waveguides has been developed and validated by data reported in literature. At the best of our knowledge, all approaches for scattering loss estimation in bent waveg- 
uides, as proposed until now, are 2D. Previously reported 3D models for scattering loss calculation were derived for straight waveguides. Moreover, these 3D models have been commonly used to estimate scattering loss in high index contrast waveguides. In this paper low index contrast guiding structures have been considered. Finally, for the first time, a closed-form expression of loss coefficient due to scattering, based on a 3D modelling, is provided in this paper.

Scattering loss suffered by a InGaAsP/InP buried waveguide and a guiding structure in silica-on-silicon technology has been calculated. These two waveguides exhibit a quite modest refractive index contrast between core and cladding materials and so, according with theoretical results in literature, we expect a quite modest scattering loss. This feature makes them particularly attractive to be adopted in the fabrication of high quality factor $(Q)$ integrated ring resonators for gyro applications, so that silica-on-silicon waveguides have been employed to realize passive integrated optical angular rate sensors based on high- $Q$ resonators [11]-[18]. The use of InGaAsP/InP buried waveguide for ring resonators realization has been firstly reported in [19]. This kind of technology exhibits the very interesting advantage to allow the monolithic integration of the ring resonator together with other active/passive integrated optical components and has enabled the fabrication of a ring resonator having a quality factor larger than 100,000 [19]. Operating wavelength $\lambda$ has been fixed in this paper as equal to $1.55 \mu \mathrm{m}$.

\section{THREE-DIMENSIONAL ALGORITHM FOR SCATTERING LOSS ESTIMATION}

Sidewall roughness induced optical loss in bent waveguides has been calculated by taking into account that, in this kind of guiding structures, electric and magnetic field components distributions are non-symmetric with respect to waveguide symmetry axis in waveguide cross section. This is the fundamental difference between straight and bent waveguides when scattering loss is to be estimated.

Each sidewall contribution to scattering loss has been separately calculated. Sidewall roughness has been modelled as a zero-mean real function $f(s)$ depending only on the curvilinear coordinate $s$ along the bent guiding structure. An exponential model has been used to write the roughness autocorrelation function $(\mathrm{ACF}) \mathrm{C}(u)$ in the form:

$$
C(u) \approx \sigma^{2} \exp \left(-\frac{|u|}{L_{C}}\right)
$$

where $\sigma$ and $L_{C}$ are the roughness standard deviation and correlation length, respectively. The exponential model for ACF has been adopted in almost all previously cited theoretical papers and it has been experimentally proved as accurate in [20]. The estimation of these two parameters is very critical for the accurate calculation of scattering loss.

According with VCM, loss coefficient $\alpha_{s c}$ (due to roughnessinduced scattering loss) in bent waveguide (whose crosssection is shown in Figure 1(a)) can be estimated by separately calculating the electromagnetic fields generated by the two

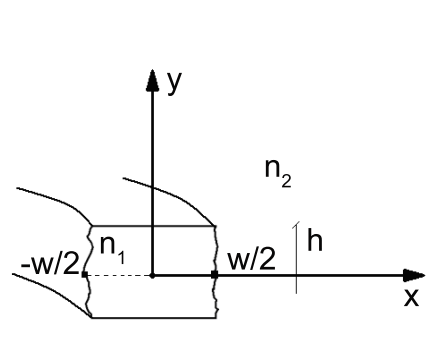

(a)

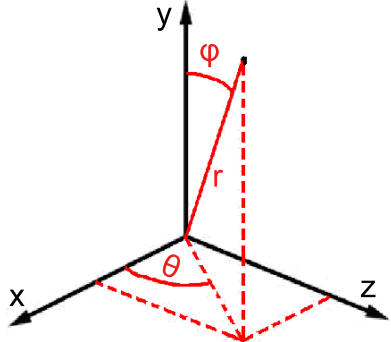

(b)
FIG. 1 (a) Bent waveguide cross-section. (b) reference system.

following equivalent current distributions (adopted reference system is illustrated in Figure 1(b))

$$
\begin{array}{lr}
\vec{J}_{1}(y)=-i \omega \varepsilon_{0}\left(n_{1}^{2}-n_{2}^{2}\right) \vec{E}\left(\frac{w}{2}, y\right) \delta(x) \delta(z) & -\frac{h}{2} \leq y \leq \frac{h}{2} \\
\vec{J}_{2}(y)=-i \omega \varepsilon_{0}\left(n_{1}^{2}-n_{2}^{2}\right) \vec{E}\left(-\frac{w}{2}, y\right) \delta(x) \delta(z) & -\frac{h}{2} \leq y \leq \frac{h}{2}
\end{array}
$$

where $\omega$ is the angular frequency of optical signal propagating within the waveguide, $\varepsilon_{0}$ is the vacuum permittivity, $\vec{E}$ is the electric field related to optical mode propagating within the waveguide, $\delta$ is the Dirac delta function and $i$ is the imaginary unit. Supposing that roughness profile of the two sidewalls are uncorrelated, loss coefficient $\alpha_{s c}$ can be written as $[3,8]$ :

$$
\begin{aligned}
\alpha_{s c}=\oiiint_{\Sigma_{1}} & \left(\vec{S}_{1} \cdot \vec{r}\right) \tilde{C}\left(\beta-n_{2} k_{0} \cos \theta\right) d S \\
& +\oiiint_{\Sigma_{2}}\left(\vec{S}_{2} \cdot \vec{r}\right) \tilde{C}\left(\beta-n_{2} k_{0} \cos \theta\right) d S
\end{aligned}
$$

where

$$
k_{0}=\frac{2 \pi}{\lambda}, \quad \beta=k_{0} n_{e f f}, \quad \vec{r}=x \hat{x}+y \hat{y}+z \hat{z},
$$

$n_{\text {eff }}$ is the mode propagating within the waveguide effective index, $\hat{x}, \hat{y}$ and $\hat{z}$ are unit vectors along $x, y$ and $z, \theta$ is the angle between $\vec{r}$ and $y$ axis (see Figure 1(b)), $\tilde{C}$ is ACF Fourier transform, $\vec{S}_{1}$ and $\vec{S}_{2}$ are Poynting vectors relevant to the electromagnetic field generated by the two current distributions in Eq. (2) (for straight waveguides the current distributions are identical and so $\vec{S}_{1}=\vec{S}_{2}$ ). Surface integrals are calculated over the two closed surfaces (usually two spheres) $\Sigma_{1}$ and $\Sigma_{2}$. Obviously, $\Sigma_{1}$ has to include $\vec{J}_{1}$ and $\Sigma_{2}$ has to include $\vec{J}_{2}$. To compute $\vec{S}_{1}$ and $\vec{S}_{2}$ we have adopted magnetic vector potential technique assuming that the medium surrounding the two current distributions is homogeneous and that $\vec{E}$ is directed along $x$ and $y$ for quasi-TE mode and quasi-TM one, respectively. These two assumptions can be considered sufficiently accurate for low index contrast waveguides.

Two loss coefficient $\alpha_{s c}$ expressions have been derived for quasi-TE and quasi-TM polarizations (see Appendix A):

$$
\begin{aligned}
\alpha_{s c, T E}=\sum_{j=1,2} U \int_{0}^{2 \pi} & \int_{0}^{\pi} \frac{1}{1+L_{C}^{2}\left(\beta-n_{2} k_{0} \cos \theta\right)^{2}} F_{j, T E}(\theta) \\
& \times\left(\cos ^{2} \theta \sin ^{2} \phi+\cos ^{2} \phi\right) \sin \theta d \theta d \phi
\end{aligned}
$$




$$
\begin{aligned}
\alpha_{s c, T M}=\sum_{j=1,2} 2 \pi U \int_{0}^{\pi} \frac{1}{1+L_{C}^{2}\left(\beta-n_{2} k_{0} \cos \theta\right)^{2}} \\
\quad \times F_{j, T M}(\theta) \sin ^{3} \theta d \theta
\end{aligned}
$$

where

$$
\begin{gathered}
U=\frac{\pi^{2} n_{2} \sigma^{2} L_{C}}{Z_{0} \lambda^{4}}\left(n_{1}^{2}-n_{2}^{2}\right)^{2} \\
F_{1, T E}(\theta)=\left|\int_{-h / 2}^{h / 2} E_{x}\left(\frac{w}{2}, y\right) e^{-j n_{2} k_{0} y \cos \theta} d y\right|^{2} \\
F_{2, T E}(\theta)=\left|\int_{-h / 2}^{h / 2} E_{x}\left(-\frac{w}{2}, y\right) e^{-j n_{2} k_{0} y \cos \theta} d y\right|^{2} \\
F_{1, T M}(\theta)=\left|\int_{-h / 2}^{h / 2} E_{y}\left(\frac{w}{2}, y\right) e^{-j n_{2} k_{0} y \cos \theta} d y\right|^{2} \\
F_{2, T M}(\theta)=\left|\int_{-h / 2}^{h / 2} E_{y}\left(-\frac{w}{2}, y\right) e^{-j n_{2} k_{0} y \cos \theta} d y\right|^{2}
\end{gathered}
$$

$\phi$ is the angle between the $x$ axis and the $\vec{r}$ projection on $x-z$ plane (see Figure $1(b))$ and $Z_{0}$ is the free space impedance. In Eq. (8), $E_{x}$ and $E_{y}$ have been calculated by $3 \mathrm{D}$ mode solver based on mode-matching method [21], whereas the four functions $F_{j, T E / T M}(j=1,2)$ have been numerically computed for $\theta$ ranging from 0 to $\pi$. $F_{j, T E / T M}(j=1,2)$ dependence on $\theta$ is quite Gaussian with the maximum value for $\theta=\pi / 2$. Finally, loss coefficients $\alpha_{T E}$ and $\alpha_{T M}$ have been estimated by numerical integrations [22].

The two closed-form expressions of loss coefficients reported in Eqs. (5) and (6) permit a quick and accurate 3D computation of scattering loss suffered by bent waveguides. Accuracy of scattering loss calculation by Eqs. (5) and (6) eminently depends on accuracy in experimental estimation of the two parameters of the ACF $\sigma$ and $L_{C}$ ). Numerical errors introduced by our modelling are surely negligible with respect to uncertainty affecting the measure of $\sigma$ and $L_{C}$.

The developed numerical algorithm for $\alpha_{s c}$ estimation has been validated (for low index contrast waveguides) by investigating the scattering loss of a straight silicon oxynitride $/ \mathrm{SiO}_{2}$ buried waveguide. Core region has been supposed to be realized by silicon oxynitride (refractive index $=1.5$ ) and its height has been fixed as $2 \mu \mathrm{m}$. Core width has been varied in the range between $2 \mu \mathrm{m}$ and $3 \mu \mathrm{m}$. We have assumed the core as completely surrounded by $\mathrm{SiO}_{2}$ (refractive index $=1.45$ ) and an operating wavelength of $1.55 \mu \mathrm{m}$. Obtained results have been compared with those reported in [8] for the same waveguide (see Figure 2). Difference between data reported in literature and results of our modelling is less than $3 \%$.

To estimate the error induced by approximations in closedform expressions of Eqs. (5) and (6), we have also estimated scattering loss of silicon oxynitride $/ \mathrm{SiO}_{2}$ waveguide by a fully-numerical technique based on Eq. (3). The electromagnetic field generated by the two current distributions in Eq. (2) has been numerically calculated by 3D FEM algorithm [23]. The two Poynting vectors relevant to this electromagnetic field and the two integrals in Eq. (3) have been numerically estimated, too. Then, in this fully-numerical estimation of scattering loss, we have removed the hypothesis of homogeneous

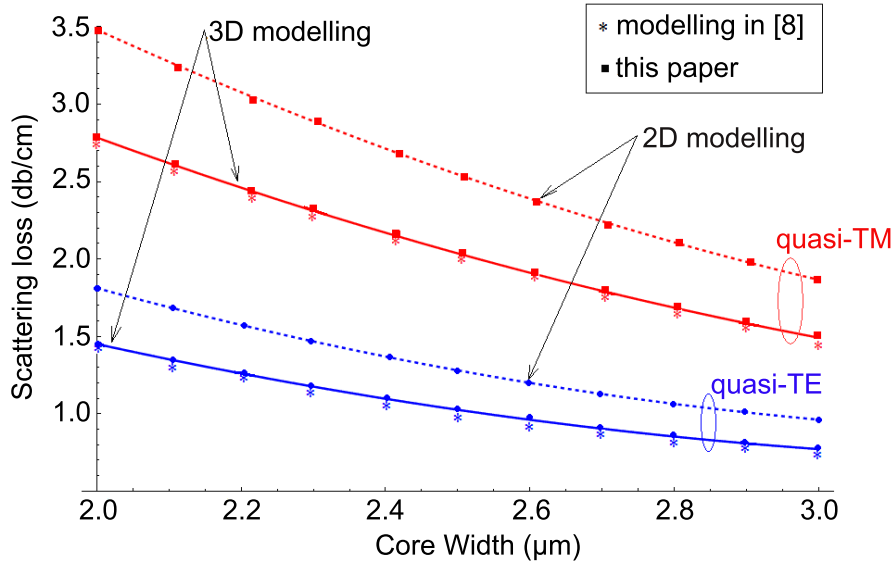

FIG. 2 Scattering loss dependence on core width for a Silicon 0xinitride/ $/ \mathrm{Si}_{2}$ waveguide calculated by $3 \mathrm{D}$ model developed in this paper, 3D model as reported in [8] and $2 \mathrm{D}$ model proposed in [4].

medium surrounding the two current distributions in Eq. (2). Results of this fully-numerical approach are practically the same (difference $<0.1 \%$ ) as those obtained by using our closed-form expressions (Eqs. (5) and (6)). This result is related to low index contrast in silicon oxynitride $/ \mathrm{SiO}_{2}$ waveguides, as occurs in all guiding structures considered in this paper. For high index contrast waveguides, we expect better accuracy of fully-numerical technique over formulas in Eqs. (5) and (6).

Scattering loss of silicon oxynitride $/ \mathrm{SiO}_{2}$ waveguide has been calculated also by the 2D approach proposed in [4] (see Figure 2). For the considered waveguide, 2D approximation induces an error around $15-20 \%$ in scattering loss estimation.

\section{SCATTERING LOSS CALCULATION}

The proposed analytical approach, described in previous paragraph, has been applied to calculate scattering loss suffered by two low index contrast waveguides which can be effectively employed to realize high $Q$ ring resonators. In particular a InGaAsP/InP buried waveguide exhibiting an index contrast $\Delta$ equal to $5.74 \%$ and a silica-on-silicon guiding structure with $\Delta=0.75 \%$ have been considered.

In Figure 3(a) the cross-section of the InGaAsP/InP waveguide is depicted. $\operatorname{In}_{0.75} \mathrm{Ga}_{0.25} \mathrm{As}_{0.55} \mathrm{P}_{0.45}$ (wavelength band-gap of $1.25 \mu \mathrm{m}$ and refractive index at operating wavelength $\left.n_{1}=3.361\right)$ has been considered for the core region. This material is assumed to be deposited on a $\mathrm{InP}\left(n_{2}=3.168\right)$ substrate and completely buried in $\operatorname{InP}\left(\operatorname{In}_{0.75} \mathrm{Ga}_{0.25} \mathrm{As}_{0.55} \mathrm{P}_{0.45}\right.$ and InP lattice constants are matched). As in [19], buried core region height and width have been fixed as $400 \mathrm{~nm}$ and $900 \mathrm{~nm}$, respectively. This waveguide supports both fundamental quasi-TE and quasi-TM modes.

Figure 3(b) shows the considered silica-on-silicon waveguide. The core region is realized by Ge doped silica (refractive index at operating wavelength $n_{1}=1.461$ ) and it is completely surrounded by silica $\left(n_{2}=1.45\right)$. Core region width and height are both equal to $6 \mu \mathrm{m}$ (as in [14]). 


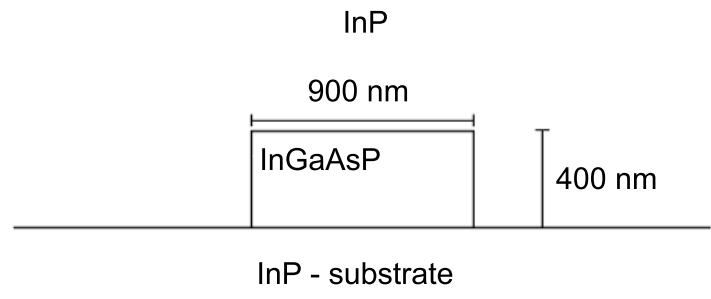

(a)

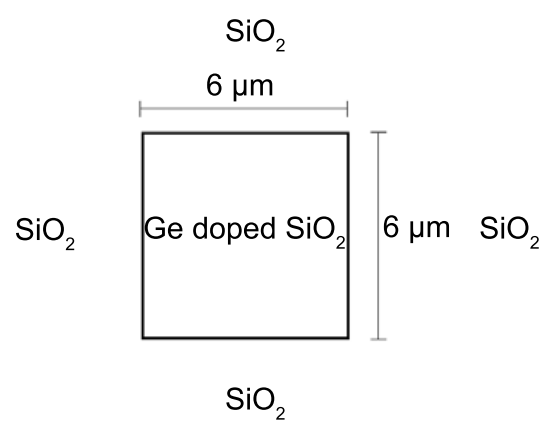

(b)

FIG. 3 (a) InGaAsP-InP buried waveguide. (b) silica-on-silicon waveguide.

Adopting our approach, the scattering loss in a $\mathrm{In}_{0.75} \mathrm{Ga}_{0.25} \mathrm{As}_{0.55} \mathrm{P}_{0.45} / \mathrm{InP}$ buried waveguide has been calculated as dependent on curvature radius $R$ (see Figure 4). Both polarizations have been considered and ACF exponential model parameters $\sigma$ and $L_{C}$ have been fixed as equal to $10 \mathrm{~nm}$ and $50 \mathrm{~nm}$, respectively. Either for quasi-TE or quasi-TM mode, scattering loss rapidly decreases (by about $20 \%$ ) when $R$ increases from $40 \mu \mathrm{m}$ to $100 \mu \mathrm{m}$. For $R$ larger than $160 \mu \mathrm{m}$, scattering loss remains quite constant. For all $R$ values, scattering loss is larger for quasi-TM mode than for quasi-TE one. This is due to the fact that in correspondence of waveguide sidewalls $E_{y}$ (for quasi-TM mode) is larger than $E_{x}$ (for quasi-TE mode). Fixing $\sigma$ equal to $10 \mathrm{~nm}$ and $R=1 \mathrm{~mm}$, correlation length influence on scattering loss has been investigated with $L_{C}$ varying from $10 \mathrm{~nm}$ to $90 \mathrm{~nm}$. As it is evident from Figure 5(a), scattering loss monotonically increases by increasing the correlation length from $10 \mathrm{~nm}$ to $90 \mathrm{~nm}$. This quite strong dependence of scattering loss on correlation length, especially for low $L_{C}$ values, shows that an experimental error in $L_{C}$ estimation may seriously affect the accuracy of scattering loss calculation.

Scattering loss dependence on operating wavelength has been investigated for $\lambda$ ranging from $1500 \mathrm{~nm}$ to $1600 \mathrm{~nm}$ (Figure 5(b)). Radius of curvature has been fixed as $1 \mathrm{~mm}$, assuming $\sigma=10 \mathrm{~nm}$ and $L_{C}=50 \mathrm{~nm}$. InP and $\mathrm{In}_{0.75} \mathrm{Ga}_{0.25} \mathrm{As}_{0.55} \mathrm{P}_{0.45}$ refractive index dependence on $\lambda$ has been taken into account by the empirical model described in [24]. Increasing operating wavelength, scattering loss decreases according with the following expression $\left(\alpha_{s c}\right.$ in $\mathrm{dB} / \mathrm{cm})$ :

$$
\alpha_{s c} \approx K \frac{1}{\lambda^{\rho}}
$$

where $K$ and $\rho$ are fitting parameters obtainable starting from numerically calculated data. The coefficient $\rho$ is equal to 4.204 and 4.722 for quasi-TE and quasi-TM, respectively. Increasing

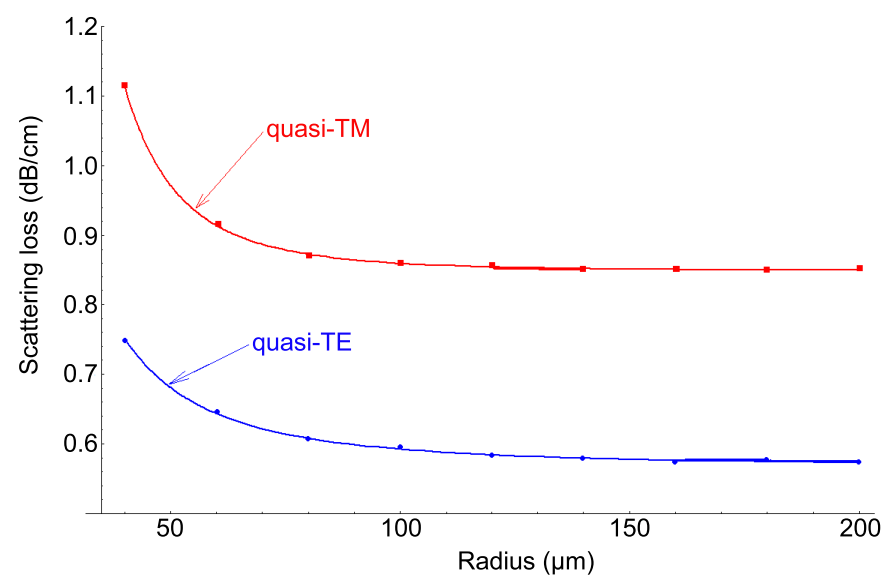

FIG. 4 Scattering loss dependence on radius of curvature $R(\lambda=1550 \mathrm{~nm}, \sigma=10 \mathrm{~nm}$ and $L_{C}=50 \mathrm{~nm}$ ).

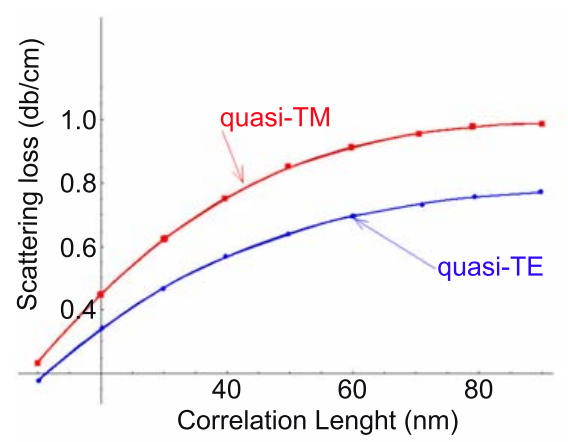

(a)

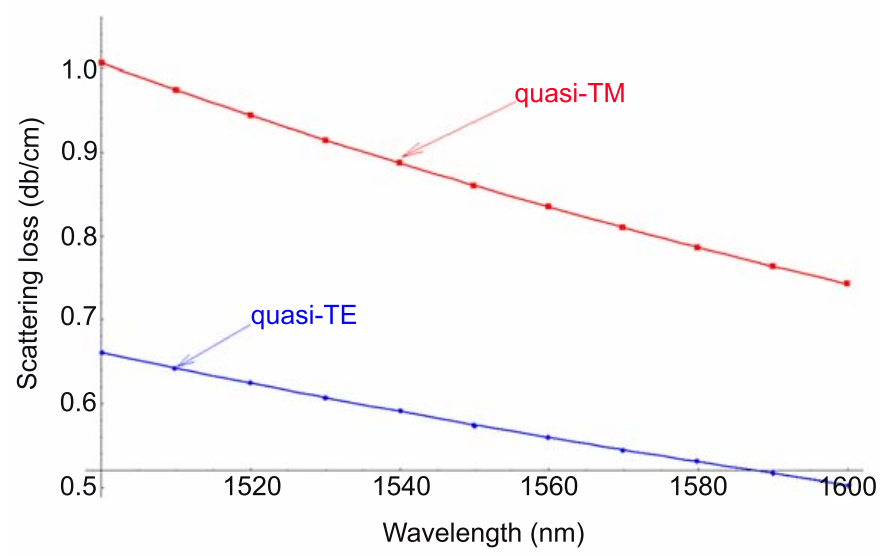

(b)

FIG. 5 (a) Scattering loss dependence on correlation length $(\lambda=1550 \mathrm{~nm}, \sigma=10 \mathrm{~nm}$ and $R=1 \mathrm{~mm}$ ) and (b) operating wavelength $\left(\sigma=10 \mathrm{~nm}, L_{C}=50 \mathrm{~nm}\right.$ and $R=1 \mathrm{~mm})$.

$\lambda$, scattering loss decreases more rapidly for quasi-TM than for quasi-TE mode $\left(\rho_{T E}<\rho_{T M}\right)$. Examining Eqs. (5), (6) and (7), we could expect $\alpha_{S C}$ as proportional to $\lambda^{-4}$ (see $U$ expression), but we have also to consider that $n_{1}, n_{2}$, and $k_{0}$ (and so also $\beta$ and $\left.F_{j, T E / T M}\right)$ also depend on $\lambda$.

Scattering loss suffered by the silica-on-silicon waveguide has been estimated assuming sidewall roughness standard deviation $\sigma$ as $200 \mathrm{~nm}$. Scattering loss dependence on curvature radius has been investigated (see Figure 6(a)) for $R$ ranging from $1 \mathrm{~mm}$ to $10 \mathrm{~mm}$, fixing $L_{C}=50 \mathrm{~nm}$. Scattering loss decreases as $R$ increases. The decrease rate is larger for $R$ around 1-2 $\mathrm{mm}$ and it becomes very low for radius values larger than 
$6 \mathrm{~mm}$. As for the InGaAsP/InP waveguide, scattering loss is larger for quasi-TM than for quasi-TE mode. For all $R$ considered values and quasi-TE mode, scattering loss is around $0.1 \mathrm{~dB} / \mathrm{cm}$. This value is the same experimentally measured in [14]. Fixing $R=10 \mathrm{~mm}$, scattering loss dependence on correlation length has been investigated too. For both polarizations, we can observe that the scattering loss increase is induced by the correlation length increase.

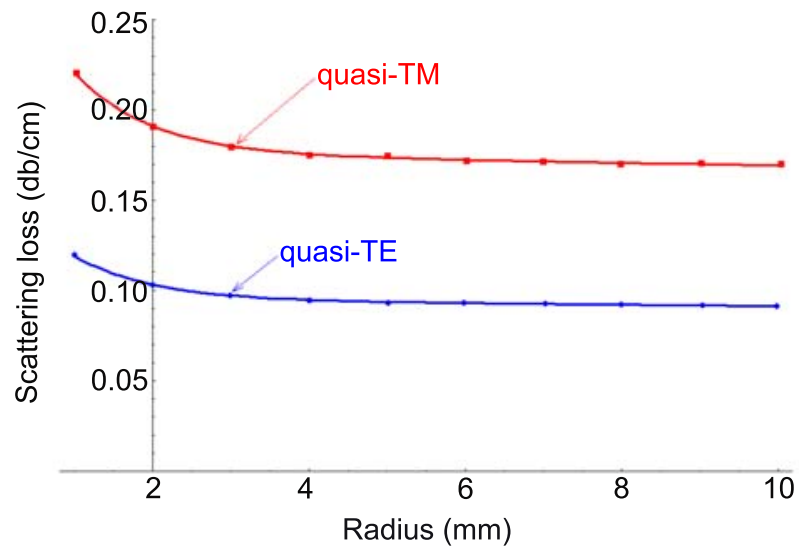

(a)

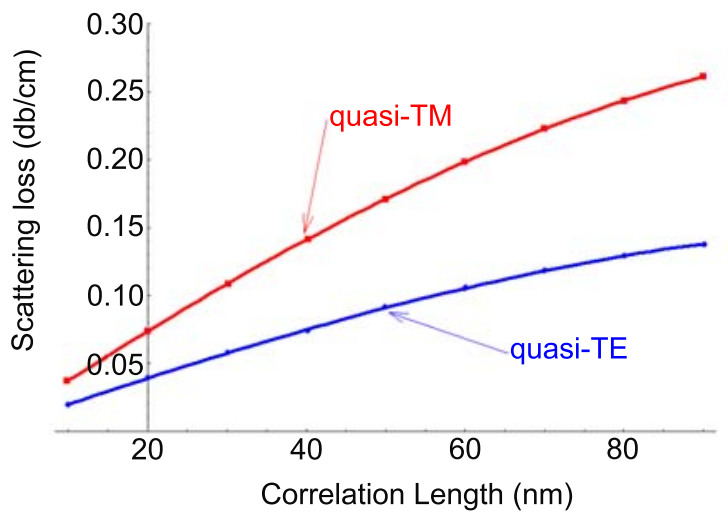

(b)

FIG. 6 (a) Scattering loss dependence on bending radius $(\lambda=1550 \mathrm{~nm}, \sigma=200 \mathrm{~nm}$ and $\left.L_{C}=50 \mathrm{~nm}\right)$ and (b) correlation length $(\lambda=1550 \mathrm{~nm}, \sigma=200 \mathrm{~nm}$, and $R=10 \mathrm{~mm}$ ).

\section{CONCLUSION}

A very general theoretical approach for scattering loss modelling in optical bent waveguides has been developed and validated. The algorithm is based on VCM and takes into account the 3D guiding structure. Two very useful closedform expressions have been derived for the calculation of scattering loss relevant to quasi-TE and quasi-TM modes. Proposed approach has been adopted to investigate scattering loss in silica-on-silicon and InGaAsP/InP waveguides. We have proved that scattering loss decreases by increasing the bending radius and decreases also when wavelength increases. silica-on-silicon waveguide having an index contrast of $0.75 \%$ exhibits scattering loss significantly lower than In$\mathrm{GaAsP} / \mathrm{InP}$ waveguide, whose index contrast is larger than $5 \%$. For quasi-TE mode, scattering loss is around $0.1 \mathrm{~dB} / \mathrm{cm}$ and $0.6 \mathrm{~dB} / \mathrm{cm}$ for silica-on-silicon and InGaAsP/InP waveguides, respectively.

\section{A APPENDIX}

In this Appendix we derive Eqs. (5) and (6) starting from current distributions in Eq. (2) and the general $\alpha_{s c}$ expression in Eq. (3). Magnetic vector potential technique has been used. In the far-field region, the general expression of magnetic vector potential $\vec{A}$ relevant to the electromagnetic field generated by a current distribution $\vec{J}$ surrounded by a homogeneous medium having a refractive index equal to $n$ is:

$$
\vec{A}=\frac{\mu_{0}}{4 \pi}\left[\frac{e^{i n k_{0} r}}{r}\right] \iiint \vec{J}\left(\vec{r}^{\prime}\right) e^{-i n k_{0}\left(\vec{r} \cdot \vec{r}^{\prime}\right)} d V^{\prime}
$$

where $\mu_{0}$ is the free space permeability, $r$ is $\vec{r}$ vector magnitude and $\vec{r}^{\prime}$ is source point position vector. The Poynting vector $\vec{S}$ relevant to $\vec{A}$ is equal to:

$$
\vec{S}=\vec{r} \frac{\omega n k_{0}}{2 \mu_{0}}|\vec{r} \times \vec{A}|^{2} .
$$

We firstly explain how to obtain $\alpha_{s c}$ for quasi-TE mode (Eq. (5)). In particular, in the sum in Eq. (5) we show how derive the first term (that relevant to $j=1$ ). The second addend is obtainable in the same manner. We have to calculate the Poynting vector $\vec{S}_{1}$ related to the electromagnetic field generated by $\vec{J}_{1}$. For quasi-TE mode, $\vec{J}_{1}$ expression is:

$$
\vec{J}_{1}(y)=-i \omega \varepsilon_{0}\left(n_{1}^{2}-n_{2}^{2}\right) E_{x}\left(\frac{w}{2}, y\right) \delta(x) \delta(z) \hat{x} .
$$

In the far-field region, the magnetic vector potential $\vec{A}_{1}$ related to the electromagnetic field generated by $\vec{J}_{1}$ is given by:

$$
\begin{aligned}
\vec{A}_{1}=\hat{x} \frac{\mu_{0}}{4 \pi} & {\left[\frac{e^{i n_{2} k_{0} r}}{r}\right]\left[-i \omega \varepsilon_{0}\left(n_{1}^{2}-n_{2}^{2}\right)\right] } \\
& \times \int_{-h / 2}^{h / 2} E_{x}\left(\frac{w}{2}, y\right) e^{-i n_{2} k_{0} y \cos \theta} d y
\end{aligned}
$$

and the relevant Poynting vector is equal to:

$$
\vec{S}_{1}=\vec{r} \frac{\omega n_{2} k_{0}}{2 \mu_{0}}\left[\left|A_{1, \theta}\right|^{2}+\left|A_{1, \phi}\right|^{2}\right]
$$

where $A_{1, \theta}$ and $A_{1, \phi}$ are $\vec{A}_{1}$ components along $\theta$ and $\phi$ (a spherical coordinate system has been considered). These two quantities can be easily calculated giving $\hat{x}$ in spherical coordinates, as:

$$
\begin{aligned}
& \left|A_{1, \theta}\right|^{2}=\left(\frac{\mu_{0}}{4 \pi r}\right)^{2}\left[-i \omega \varepsilon_{0}\left(n_{1}^{2}-n_{2}^{2}\right)\right]^{2} F_{1, T E}(\theta)(\cos \theta \sin \phi)^{2} \\
& \left|A_{1, \phi}\right|^{2}=\left(\frac{\mu_{0}}{4 \pi r}\right)^{2}\left[-i \omega \varepsilon_{0}\left(n_{1}^{2}-n_{2}^{2}\right)\right]^{2} F_{1, T E}(\theta)(\cos \phi)^{2} .
\end{aligned}
$$

Then, we have that the two terms in the first integral of Eq. (3) are equal to:

$$
\begin{gathered}
\left(\vec{S}_{1} \cdot \vec{r}\right)=\frac{\omega n_{2} k_{0}}{2 \mu_{0}}\left(\frac{\mu_{0}}{4 \pi r}\right)^{2} \omega^{2} \varepsilon_{0}^{2}\left(n_{1}^{2}-n_{2}^{2}\right)^{2} F_{1, T E}(\theta) \\
\times\left[(\cos \theta \sin \phi)^{2}+(\cos \phi)^{2}\right] \\
\tilde{C}\left(\beta-n_{2} k_{0} \cos \theta\right)=\frac{2 \sigma^{2} L_{C}}{1+L_{C}^{2}\left(\beta-n_{2} k_{0} \cos \theta\right)^{2}} .
\end{gathered}
$$


Remembering that the infinitesimal spherical surface element is:

$$
d S=r^{2} \sin \theta d \theta d \phi
$$

we have the first integral in Eq. (3) in the form:

$$
\begin{aligned}
& \int_{0}^{2 \pi} \int_{0}^{\pi} \frac{2 \sigma^{2} L_{C}}{1+L_{C}^{2}\left(\beta-n_{2} k_{0} \cos \theta\right)^{2}} \frac{\omega n k_{0}}{2 \mu_{0}}\left(\frac{\mu_{0}}{4 \pi r}\right)^{2} \omega^{2} \varepsilon_{0}^{2}\left(n_{1}^{2}-n_{2}^{2}\right)^{2} \\
& \left.\quad \times F_{1, T E}(\theta)\left[(\cos \theta \sin \phi)^{2}+(\cos \phi)^{2}\right] r^{2} \sin \theta d \theta d \phi . \quad \text { (A. } 11\right)
\end{aligned}
$$

Eq. (A.11) is equivalent to the first term in the sum of Eq. (5).

The first term in the sum of Eq. (6) ( $\alpha_{s c}$ for quasi-TM mode) can be derived by a similar procedure. In particular, we have that:

$$
\begin{aligned}
& \vec{J}_{1}(y)=-i \omega \varepsilon_{0}\left(n_{1}^{2}-n_{2}^{2}\right) E_{y}\left(\frac{w}{2}, y\right) \hat{y} \\
& \vec{A}_{1}= \hat{y} \frac{\mu_{0}}{4 \pi}\left[\frac{e^{i n_{2} k_{0} r}}{r}\right]\left[-i \omega \varepsilon_{0}\left(n_{1}^{2}-n_{2}^{2}\right)\right] \\
& \quad \times \int_{-h / 2}^{h / 2} E_{y}\left(\frac{w}{2}, y\right) e^{-i n_{2} k_{0} y \cos \theta} d y \\
& \vec{S}_{1}= \vec{r} \frac{\omega n_{2} k_{0}}{2 \mu_{0}}\left[\left|A_{1, \theta}\right|^{2}\right] \\
&\left|A_{1, \theta}\right|^{2}=\left(\frac{\mu_{0}}{4 \pi r}\right)^{2}\left[-i \omega \varepsilon_{0}\left(n_{1}^{2}-n_{2}^{2}\right)\right]^{2} F_{1, T M}(\theta)(\sin \theta)^{2} \\
&\left(\vec{S}_{1} \cdot \vec{r}\right)= \frac{\omega n_{2} k_{0}}{2 \mu_{0}}\left(\frac{\mu_{0}}{4 \pi r}\right)^{2} \omega^{2} \varepsilon_{0}^{2}\left(n_{1}^{2}-n_{2}^{2}\right)^{2} \\
& \quad \times F_{1, T M}(\theta)(\sin \theta)^{2}
\end{aligned}
$$

Starting from the expression in Eq. (A.16) and observing that $\tilde{C}$ formula remains unaltered, we obtain the first term in Eq. (6). Similarly, the second addend in Eq. (6) can be derived.

\section{ACKNOWLEDGEMENTS}

The work has been partially funded by European Space Agency (ESA) under IOLG project n. 1678/02/NL/PA, in the frame of ESA-Politecnico di Bari agreement $n$. 20199/06/NL/PA. Useful discussions with E. Armandillo from ESA are acknowledged.

\section{References}

[1] C. Ciminelli, F. Dell'olio, C. E. Campanella, V. M. N. Passaro, and M. N. Armenise, "Integrated Optical Ring Resonators: Modelling and Technologies" in Progress in Optical Fibers, P. S. Emersone, ed. (NOVA Science Publishers, New York, 2009).

[2] D. Marcuse, "Mode conversion caused by surface imperfections of a dielectric slab waveguide" ATRT Tech. J. 48, 3187-3215 (1969)

[3] J. P. R. Lacey and F. P. Payne, "Radiation loss from planar waveguides with random wall imperfections" Pr. Inst. Electr. Elect. 137, 282-288 (1990).

[4] F. P. Payne and J. P. R. Lacey, "A theoretical analysis of scattering loss from planar optical waveguides" Opt. Quant. Electron. 26, 977-986 (1994).

[5] K. K. Lee, D. R. Lim, H. C. Luan, A. Agrawal, J. Foresi, and L. C. Kimerling, "Effect of size and roughness on light transmission in a
Si/SiO2 waveguide: Experiments and model" Appl. Phys. Lett. 77, 1617-1619 (2000).

[6] F. Grillot, L. Vivien, S. Laval, D. Pascal, and E. Cassan, "Size influence on the propagation loss induced by sidewall roughness in ultrasmall SOI waveguides" IEEE Photonic. Tech. L. 16, 1661-1663 (2004).

[7] C. Poulton, C. Koos, M. Fujii, A. Pfrang, T. Schimmel, J. Leuthold, and W. Freude, "Radiation modes and roughness loss in high index-contrast waveguides" IEEE J. Sel. Top. Quant. 12, 1306-1321 (2006).

[8] T. Barwicz and H. A. Haus, "Three-dimensional analysis of scattering losses due to sidewall roughness in microphotonic waveguides" J. Lightwave Technol. 23, 2719-2732 (2005).

[9] B. E. Little and S. T. Chu, "Estimating surface-roughness loss and output coupling in microdisk resonators" Opt. Lett. 21, 1390-1392 (1996).

[10] M. N. Armenise, V. M. N. Passaro, F. De Leonardis, and M. Armenise, "Modeling and Design of a Novel Miniaturized Integrated Optical Sensor for Gyroscope Applications" J. Lightwave Technol. 19, 1476-1494 (2001).

[11] C. Ciminelli, F. Peluso, E. Armandillo, and M. N. Armenise, Modeling of a new integrated optical angular velocity sensor (Optronics Symposium (OPTRO), Paris, 8-12 May 2005).

[12] C. Ciminelli, F. Peluso, and M. N. Armenise, "A new integrated optical angular velocity sensor" Proc. SPIE 5728, 93-100 (2007).

[13] C. Ciminelli, F. Peluso, N. Catalano, B. Bandini, E. Armandillo, and M. N. Armenise, Integrated optical gyroscope using a passive ring resonator $\left(5^{\text {th }}\right.$ Round Table on Micro/Nano Technologies for Space, Noordwijk, 3-5 October 2005).

[14] C. Ciminelli, Innovative photonic technologies for gyroscope systems (EOS Topical Meeting - Photonic Devices in Space, Paris, 18-19 October 2006).

[15] C. Ciminelli, C. E. Campanella, and M. N. Armenise, Design of passive ring resonators to be used for sensing applications 278-280 (First Mediterranean Photonics Conference, Ischia, 2008).

[16] C. Ciminelli, C. E. Campanella, and M. N. Armenise, "Optimized Design of Integrated Optical Angular Velocity Sensors based on a Passive Ring Resonator" to be published in J. Lightwave Technol. (2009).

[17] K. Suzuki, K. Takiguchi, and K. Hotate, "Monolithically Integrated Resonator Microoptic Gyro on Silica Planar Lightwave Circuit" J. Lightwave Technol. 18, 66-72 (2000).

[18] H. Ma, X. Zhang, Z. Jin, and C. Ding, “Waveguide-type optical passive ring resonator gyro using phase modulation spectroscopy technique" Opt. Eng. 45, 080506 (2006).

[19] S. J. Choi, K. Djordjev, Z. Peng, Q. Yang, S. J. Choi, and P. D. Dapkus, "Laterally Coupled Buried Heterostructure High- $Q$ Ring Resonators" IEEE Photonic. Tech. L. 16, 2266-2268 (2004).

[20] T. Barwicz, and H. I. Smith, " Evolution of line-edge roughness during fabrication of high-index-contrast microphotonic devices" J. Vac. Sci. Technol. B 21, 2892-2896 (2003).

[21] Fimmwave 4.6, Photon Design (2007).

[22] Mathematica 7, Wolfram Research (2007).

[23] Comsol Multiphysics 3.2, COMSOL (2005).

[24] F. F. Soares, Photonic integrated true-time-delay beamformers in InP technology paragraph 2.3 (Ph.D thesis, Technische Universiteit Eindhoven, 2006). 